\title{
The One-Dimensional Response of a Water-Filled Double Hull to Underwater Blast: Experiments and Simulations
}

\author{
A. Schiffer ${ }^{*}$ and V.L. Tagarielli \\ Department of Aeronautics, Imperial College London, SW5 2AZ, UK
}

\begin{abstract}
Laboratory-scale fluid-structure interaction (FSI) experiments and finite element (FE) simulations are performed to examine the one-dimensional blast response of double-walled hulls, consisting of two skins sandwiching a layer of water. Both monolithic and sandwich designs are considered for the outer skin. Experiments are conducted in a transparent shock tube which allows measurements of water cavitation and hull response by high-speed photography. Experiments and FE predictions are found in good agreement and allow concluding that the impulse imparted to double hulls by underwater explosions can be dramatically reduced by employing the sandwich construction of the outer skin; such reductions are scarcely sensitive to the thickness of the water layer.
\end{abstract}

Keywords: fluid-structure interaction, cavitation, sandwich plate, shock wave, finite element Submitted to Journal of Impact Engineering, May 2013

\footnotetext{
* Corresponding author: Tel. +44(0)2075 945129, e-mail address: a.schiffer@imperial.ac.uk
} 


\section{INTRODUCTION}

Hulls of underwater vessels may consist of an inner pressure hull encapsulated in an outer skin structure; the gap between these hulls may be flooded with water to control buoyancy. Understanding the response of such structures to underwater explosions is of crucial importance in defence applications; the lack of scientific publications on this subject motivates the present work.

The detonation of an explosive charge in water gives rise to spherical shock waves, travelling in water at approximately sonic speed [1]. At sufficient distance from the point of detonation, such waves can be treated as planar and the pressure history associated to the passage of this wave at a fluid particle can be described by an exponentially decaying pressure versus time pulse

$$
p(t)=p_{0} \exp (-t / \theta)
$$

with peak pressure and decay time depending on the mass and type of explosive as well as on the distance from the detonation point [2].

In order to design structural components against underwater blast, fluid-structure interaction (FSI) needs to be analysed. Pioneering studies on FSI date back to World War II; Taylor [3] analysed the response of a rigid, free-standing plate loaded by an underwater shock wave (eq. (1)) and concluded that the momentum transmitted to the plate can be reduced by decreasing the plate's mass, owing to the occurrence of cavitation in the water next to the fluid-structure interface. Valuable insight into the evolution of such cavitation phenomena is given in Kennard [4]. He found that when the pressure drops below the cavitation limit at a point in the fluid, two 'breaking fronts' start propagating from this point in opposite directions, forming an expanding pool of cavitated fluid. Subsequently, such breaking fronts can arrest, invert their motion and become 'closing fronts', reducing the volume of cavitated 
fluid. The evolution of such cavitation fronts depends on the hydrostatic fluid pressure and the characteristics of the blast wave, as well as on structural response.

During the last two decades extensive research was conducted to assess the advantage of replacing monolithic structures by sandwich constructions of equivalent mass. Several numerical studies have demonstrated that sandwich plates can outperform monolithic designs of equal mass [5-9]. The role of FSI in the response of sandwich plates subject to underwater blast has been investigated in more detail by Deshpande and Fleck [10] and Hutchinson and Xue [11]. They found that the onset of the cavitation process is located at a finite distance from the fluid-structure interface and therefore assumed that a layer of water attaches to the front face sheet, resulting in additional impulse imparted to the sandwich. Later, Liang et al. [5] and McMeeking et al. [12] developed more detailed models for underwater blast loading of sandwich plates by including the effects of the collapse of the cavitation zone.

Recently, Schiffer et al. [13] examined the effect of a non-vanishing initial hydrostatic pressure in the water upon the blast response of a rigid plate supported by a linear spring; they concluded that FSI is extremely sensitive to initial pressure in the fluid. These models accurately capture the emergence and propagation of breaking fronts and closing fronts, as outlined in [4], without using simplifying assumptions.

Early experimental work on underwater blast loading was published by the U.S. Office of Naval Research (ONR) [14]. Several studies focused on dynamic deformation and failure modes of real-size naval structures subject to blast loading in explosive test facilities [15-17]. In order to reduce the time and cost required for large-scale tests, experimental methods at laboratory scale have since been developed. Deshpande et al. [18] designed an experimental apparatus able to generate realistic underwater blast waves in a water-filled steel shock tube 
and used it to simulate 1D blast loading of monolithic plates and foam-cored sandwich panels. Subsequently, McShane et al. [19] employed this underwater shock simulator [18] to perform blast loading on free-standing sandwich plates with metallic lattice cores, measuring transmitted momentum and permanent core compression and providing experimental evidence for the benefits offered by the sandwich construction in terms of blast performance. Espinosa et al. [20] followed a similar approach and designed a divergent shock tube to measure dynamic deformation of clamped plates subject to underwater blast loading. This apparatus was then employed by [21-23] to study failure modes and damage mechanisms exhibited by monolithic plates and sandwich panels in an underwater blast event. Wadley et al. [24] performed underwater blast loading on sandwich specimens by using an underwater explosive test rig comprising a water-filled cardboard cylinder placed on a recessed steel plate in which the specimen was located, capable of measuring the loads transmitted to the supports in a blast event.

Recent experimental work on FSI in underwater blast was carried out by Schiffer and Tagarielli [25], who developed an experimental apparatus able to reproduce blast loading in initially pressurised water, in order to simulate blast loading in deep water. The apparatus consists of a shock tube similar to that used in [18] but made from a transparent material, to allow observation of specimen motion and cavitation phenomena in the water.

Subsequently, this apparatus [25] was used by Schiffer and Tagarielli [26] to examine the 1D response of water-backed and air-backed sandwich plates to blast in deep or shallow water, measuring the propagation velocity of cavitation fronts as well as core crush and impulse imparted to the sandwich, and providing experimental evidence for theoretical predictions [4, $5,10,11]$ in the process. 
Double-walled hull construction complicates the prediction of structural loading consequent to underwater blast, as pressure waves travelling in the water layer between the two hulls can significantly affect the ensuing cavitation process as well as the overall structural response; such phenomena need to be thoroughly understood. In this study we employ the apparatus developed in [25] in order to investigate the response of water-filled double hulls subject to underwater blast. In the process, we consider two practical design concepts: (i) double hulls with monolithic skins and (ii) double hulls with monolithic inner skin and sandwich outer skin. High-speed photography is employed to observe the dynamic structural response as well as cavitation processes in the water. Dynamic fluid pressure measurements serve to deduce the impulse imparted to the inner hull and to explore its sensitivity to the thickness of the water layer.

The outline of this paper is as follows: in Section 2 we define the problems under investigation; in Section 3 we briefly describe the laboratory setup, specimen manufacture and we outline the experimental programme, while details on the FE simulations are given in Section 4; results obtained from the experiments and FE calculations are presented and discussed in Section 5 and we summarise the main findings of this study in Section 6.

\section{PROBLEM DEFINITION}

In this study, the response of double hulls subject to underwater blast is examined. Two different design concepts are considered and described below.

\section{a) Monolithic double hull.}

The schematic in Fig. 1a illustrates the geometry of a double hull consisting of two rigid plates separated by a water layer of thickness $D$. We define as 'outer skin' the plate in 
contact with water on both sides while the underlying inner structure is termed as 'inner hull' and is in contact with water on the front face only. The outer skin (of mass per unit area $m_{F}$ ) is loaded on the outer surface by an exponentially decaying shock wave (eq. (1)) of peak pressure $p_{0}$ and decay time $\theta$. Subsequently, pressure waves transmitted into the water layer impinge on the inner hull (of mass per unit area $m_{B}$ ).

\section{b) Sandwich double hull.}

The problem geometry for a sandwich double hull is sketched in Fig. 1b. It comprises of a monolithic inner hull (areal mass $m_{B}$ ) and an outer skin of sandwich construction (areal mass $m_{F}$ ), with a water layer (thickness $D$ ) located between outer skin and inner hull; the loading case is taken as described above for monolithic double hulls.

For each of the above design concepts, two different boundary conditions are considered:

(i) Stationary inner hull: the outer skin is free to move while the motion of the inner hull is constrained, representing the case of a double hull with $m_{B} \rightarrow \infty$.

(ii) Unsupported inner hull: both outer skin and inner hull are unsupported and $m_{B} \approx m_{F}$.

\section{FLUID-STRUCTURE INTERACTION EXPERIMENTS}

\subsection{Laboratory setup and instrumentation}

Underwater shock experiments were performed using the shock tube developed by Schiffer and Tagarielli [25], schematically illustrated in Fig. 2a. The outer skin specimen was inserted into the shock tube, approximately $200 \mathrm{~mm}$ from the distal end, and two water columns were located in the tube on both sides of the specimen (see Fig. 2a). The tube was capped at the front end with a sealing nylon piston and at the distal end with the inner hull specimen; bleed 
valves were included in front piston and inner hull specimen to evacuate air-bubbles trapped in the water columns prior to the dynamic experiment. The time histories of the interface pressure applied on the wet face of the inner hull were measured using a piezoelectric pressure gauge (PCB Piezoelectronics Inc., type 113B23) mounted centrally on the inner hull specimen and flush to the specimen front face. For experiments performed with stationary inner hull specimens, motion of the inner hull specimen was mechanically constrained by an anvil and a distance bush, as shown in Fig. 2a.

Exponentially decaying shock waves according to eq. (1) were generated in the tube by firing a steel striker of diameter $d_{s}=18.5 \mathrm{~mm}$ at the front piston (Fig. 2a), as described in [18]. The incident pressure wave was measured using a piezoelectric pressure gauge (PCB Piezoelectronics Inc., type 113B23) at a distance approximately $120 \mathrm{~mm}$ from the front end.

\subsection{Specimen design and manufacture}

\subsubsection{Outer skin specimens}

The specimen geometry of a monolithic outer skin specimen is sketched in Fig. 2b. Round specimens were machined from aluminium to diameter $d=27 \mathrm{~mm}$ and thickness $h$; two circular grooves were included for fitting O-rings on the specimens.

In the case of sandwich construction, the outer hull comprised a crushable foam core encased between metallic face sheets of equal thickness $h_{f}$, diameter $d_{f}$ and areal mass $m_{f}$, see Fig. 2c. The sandwich cores were machined from polymer foam (Rohacell) to diameter $d_{c}$ and thickness $c$. Rohacell foams are closed cell foams made from polymetacrylimide (PMI) and are available in a range of densities [27]. In this study, three different foam densities are considered, $\rho_{c}=71 \mathrm{kgm}^{-2}, \rho_{c}=110 \mathrm{kgm}^{-2}$ and $\rho_{c}=200 \mathrm{kgm}^{-2}$, and details on mechanical 
properties [26, 27], thickness and diameter of the chosen sandwich core designs are listed in Table 1. The face sheets were machined from aluminium to diameter $d_{f}=27 \mathrm{~mm}$ and thickness $h_{f}=8 \mathrm{~mm}$, fitted with O-rings and bonded to the sandwich core using epoxy adhesive; the total masses of the obtained sandwich specimens were in the range $m_{F}=41.9-42.7 \mathrm{kgm}^{-2}$.

\subsubsection{Inner hull specimens}

In all experiments performed in this study, monolithic (rigid) inner hull specimens were used and their geometry was similar to that sketched in Fig. 2b but with a threaded hole included at the centre for mounting the pressure sensor. In addition, they were machined with a bleed valve (out of centre) to evacuate air-bubbles trapped in the water layer (see Fig. 2a).

\subsection{Outline of the experimental study}

The peak impulse $I_{B, \max }=\max \left[I_{B}(t)\right]$ imparted to the inner hull in a blast event is indicative of the severity of structural loading, while the core crush $\varepsilon_{c}^{p}$ partly determines to what extent the structural integrity of a sandwich hull is maintained after blast loading. The objective of this study is to determine, for both monolithic and sandwich designs, the sensitivity of impulse $I_{B, \max }$ and core crush $\varepsilon_{c}^{p}$ (of sandwich hulls) to the thickness of the water layer, and to determine the benefits of the sandwich construction. To achieve this, the following test series were performed:

Test series 1 was conducted to establish, for both sandwich and monolithic double hull designs with stationary inner hulls (i.e. mimicking the case $m_{B} \rightarrow \infty$ ), the sensitivity of $I_{B \text {,max }}$ and $\varepsilon_{c}^{p}$ to the water layer thickness $D$, holding the outer skin mass $m_{F}$ as well as the decay 
time of the loading shock wave fixed, $m_{F}=42.5 \pm 0.5 \mathrm{kgm}^{-2}, \theta=0.14 \mathrm{~ms}$, respectively. Underwater shock experiments were performed on a monolithic outer skin specimen located at distances $30 \mathrm{~mm}, 80 \mathrm{~mm}, 120 \mathrm{~mm}$ and $160 \mathrm{~mm}$ from the fluid-structure interface of the fully clamped inner hull specimen (see Fig. 2a) and were then repeated by replacing the monolithic outer skin specimen with a sandwich specimen (specimen 2, see Table 1).

Test series 2 was performed to probe the effect of the sandwich core strength $\sigma_{c}$ on imparted impulse $I_{B, \max }$ and core crush $\varepsilon_{c}^{p}$ for the case $m_{B} \rightarrow \infty$. Three different types of core were considered and their properties and dimensions are listed in Table 1; the water layer thickness, the areal mass of the sandwich as well as the decay time of the loading pressure pulse were kept constant, $D=120 \mathrm{~mm}, m_{F}=42.5 \pm 0.5 \mathrm{kgm}^{-2}, \theta=0.14 \mathrm{~ms}$, respectively. Note that in both test series 1 and 2 the numerical values of the FSI parameter [3]

$$
\psi_{F}=\frac{\rho_{w} c_{w} \theta}{m_{F}}
$$

related to the areal mass $m_{F}$ of the outer skin specimen $\left(m_{F}=2 m_{f}+m_{c}\right.$ for sandwich plates) were approximately constant, $\psi_{F} \cong 3.4$, representative of the case of blast loading on largescale structures.

Test series 3 was similar to test series $1\left(m_{F}=42.5 \mathrm{kgm}^{-2}, \theta=0.14 \mathrm{~ms}, \psi_{F} \cong 3.4\right)$ but with the inner hull specimen free to move in the shock tube, i.e. simulating blast loading of double hulls with $m_{B} \approx m_{F}$. Experiments for this test series were performed with outer skin specimens made from either rigid plates or sandwich plates (specimen 1, see Table 1) and monolithic inner hull specimens $\left(m_{B}=80.2 \mathrm{kgm}^{-2}\right)$, with $\psi_{B}=\rho_{w} c_{w} \theta / m_{B}=1.8$ the FSI parameter associated to the mass of the inner hull $m_{B}$; the thickness of the water layer $D$ was adjusted to vary between $30 \mathrm{~mm}$ and $120 \mathrm{~mm}$. 


\section{FINITE ELEMENT MODELS}

One-dimensional FE simulations were performed in ABAQUS/Explicit [28] in order to compare with measurements. FE simulations also provide insight into the loading histories applied on the outer skin in a blast event which could not be directly measured with the present technique. The FE model employed to simulate blast loading of a sandwich double hull is sketched in Fig. 3. It consisted of a front water column, a water layer, a rigid inner hull plate and a sandwich plate representing the outer skin, all of which of unit width and height $H_{f}$. The front water column was chosen sufficiently long to guarantee that the FSI phase had finished before pressure wave reflections at the free end could reach the structure. The sandwich plate comprised a foam core of thickness $c$, encased between two face sheets, each of thickness $h_{f}$. The fluid columns, the face sheets and the sandwich core were tied at their interfaces and discretised by using four-noded, plane-strain quadrilateral elements with reduced integration (type CPE4R in ABAQUS). The FE models for monolithic double hulls followed a similar scheme with sandwich core and back face sheet absent.

To model fluid behaviour in ABAQUS/Explicit a Mie-Gruneisen equation of state with a linear Hugoniot relation [28] was employed in order to introduce a linear-elastic relationship between fluid pressure and volumetric strain. Density of water was taken as $\rho_{w}=1000 \mathrm{kgm}^{-3}$ and the shear modulus was set to zero. It merits comment that, in the experiments, mechanical coupling between the tube and the water column reduces the speed at which pressure pulses propagate in the fluid, as discussed in [25]; such coupling phenomena also affects the values of peak pressure $p_{0}$ and decay time $\boldsymbol{\theta}$, and therefore all simulations reported herein were performed using the measured wave speed $c_{w}=1053 \mathrm{~ms}^{-1}$ [25]. Assuming that the water cavitates when the absolute fluid pressure drops to zero (i.e. neglecting the vapour pressure of 
water, $p_{v}=2.3 \mathrm{kPa}$ at $20^{\circ} \mathrm{C}$ ) and considering an ambient pressure of $1 \mathrm{~atm}$, cavitation was modelled by setting the tensile pressure cut-off in the equation of state model [28] to $1 \mathrm{~atm}$ ( $\hat{=} 0.1 \mathrm{MPa}$ ), thus allowing the water to sustain tension up to $p=-0.1 \mathrm{MPa}$ without cavitation. In order to capture the pressure shock front with adequate accuracy, the element size of the fluid mesh was set to $0.1 \mathrm{~mm}$ in direction of wave propagation, while one single element was used along the height of the water columns.

The aluminium face sheets were modelled as linear-elastic solids with density $\rho_{f}=2700 \mathrm{kgm}^{-3}$, Young's modulus $E_{f}=69 \mathrm{GPa}$ and Poisson's ratio $v_{f}=0.3$; to discretise the face sheets, an element size of $0.3 \mathrm{~mm}$ was used along the through-thickness direction.

For the foam materials (see Table 1) the following constitutive description was used. The elastic response of the foams was taken as isotropic with density $\rho_{c}$, Young's modulus $E_{c}$, Poisson's ratio $v_{c}=0.1$ and yield stress $\sigma_{c}$. Numerical values of $\rho_{c}, E_{c}$ and $\sigma_{c}$ for each foam material are listed in Table 1 . The compressive plastic collapse of the foam was modelled using the 'crushable foam' plasticity model with isotropic hardening [29] available in ABAQUS [28]. Upon assuming normality of plastic flow to the yield surface, the yield ratio $k_{Y}=\sigma_{c}^{0} / p_{c}^{0}$ ( $\sigma_{c}^{0}$ denotes the initial yield stress in uniaxial compression and $p_{c}^{0}$ the yield stress in hydrostatic compression) can be written in terms of the plastic Poisson's ratio $v_{p}$

$$
k_{Y}=\sqrt{3\left(1-2 v_{p}\right)} .
$$

For the foams considered in this study $v_{p}=0$, thus $k_{Y}=\sqrt{3}$. The hardening curves in static compression were specified in accordance to the results of quasi-static compression tests reported in [26]. 
The strain rate dependency of the foam materials is modelled as follows. Dynamic experiments on Rohacell foams [30] have shown that the dependence of the foam's yield stress on the strain rate can be neglected. However, when a foam material is subject to intense shock loading, the compressive collapse entails propagation of a plastic shock wave, introducing additional energy dissipation mechanisms. As in [31] and [32], we introduce linear strain rate dependency in the constitutive model by employing an overstress viscoplastic model

$$
\dot{\varepsilon}^{p l}=\left(\frac{\bar{\sigma}_{c}-\sigma_{c}}{\eta}\right)
$$

where $\sigma_{c}$ is the static compression yield stress, $\bar{\sigma}_{c}$ is the yield stress at nonzero strain rate and $\eta$ is the viscosity. The effect of eq. (4) is to create a plastic shock of finite width $l_{s}$, such that conservation of energy is satisfied across the shock [31]; then, the viscosity (eq. (4)) can be calculated as

$$
\eta=\frac{l_{s} \rho_{c} \Delta v}{\varepsilon_{D}}
$$

Foam density and densification strain, $\rho_{c}$ and $\varepsilon_{D}$, respectively, were taken from Table 1 and the shock width $l_{s}$ was assumed to be on the order of the foam's pore size (Table 1). As in [26] the velocity jump $\Delta v$ across the plastic shock was approximated by the velocity imparted to a free-standing plate [3] of mass equal to that of the front face sheet alone

$$
v_{f, 0}=\frac{2 p_{0} \theta \psi_{f}^{\psi_{f} /\left(1-\psi_{f}\right)}}{m_{f}}
$$

with $\psi_{f}=\rho_{w} c_{w} \theta / m_{f}$ a non-dimensional parameter related to the areal mass of the front face sheet $m_{f}$ of the outer sandwich hull. To implement eq. (4) in the FE model, the CowperSymonds overstress power-law available in ABAQUS [28] was employed. To ensure accurate resolution of the stress and strain gradients across the plastic shock wave, an element size of $l_{s} / 12$ was chosen to discretise the foam core in through-thickness direction. 
The boundary conditions were taken as follows. As shown in Fig. 3, the transverse displacements of all nodes were constrained to zero. For FE calculations corresponding to experiments performed with stationary inner hulls, longitudinal motion of the inner hull plate was also constrained to zero. Underwater blast loading was modelled by imposing an exponentially decaying pressure boundary condition in accordance to eq. (1) at the free end of the front water column (Fig. 3).

The presence of bulk viscosity in ABAQUS/Explicit [28] can lead to significant artificial energy dissipation due to the large volumetric straining of the foam core and therefore both the linear and the quadratic bulk viscosity parameters in the core were set to zero, while they were reduced to $20 \%$ of the default values [28] in the fluid columns and the face sheets.

\section{RESULTS AND DISCUSSION}

We proceed to present observations and results obtained with the shock tube apparatus. First, we present high-speed photographs recorded in selected experiments and describe the observed cavitation phenomena. Then, we examine the measured time histories of imparted impulse $I_{B}(t)$ and core compression $\varepsilon_{c}(t)$ and we compare the obtained measurements with dynamic FE results. Finally, the measurements and FE predictions are used to explore the sensitivity of peak impulse $I_{B \text {, max }}$ and core crush $\varepsilon_{c}^{p}$ to the thickness of the water layer.

\subsection{Fluid response}

\subsubsection{Stationary inner hull $\left(m_{B} \rightarrow \infty\right)$}

In Fig. 4 we present a sequence of high-speed photographs recorded in an experiment performed to reproduce blast loading of monolithic double hulls with $m_{B} \rightarrow \infty$ (test series 1 , 
see Section 3.3). In Fig. 4a, the incident wave ( $p_{0}=11.6 \mathrm{MPa}, \theta=0.14 \mathrm{~ms}$ ), travelling from the right to the left in this figure, reaches the front face of the outer skin specimen (areal mass $m_{F}=43.0 \mathrm{kgm}^{-2}$ ). Subsequently, this specimen accelerates (Fig. 4b) and a compressive wave emanates from its back face, travelling in the water layer (of thickness $D=120 \mathrm{~mm}$ ) towards the inner hull, impinging on its stationary wet face and reflecting back into the water. This reflected wave reaches the back face of the outer skin at a later time, $t=2 \mathrm{D} / c_{w}$, affecting its response and promoting reverse motion of this specimen, see Fig. 4c. The high-speed photographs of Fig. 4 show that cavitation did not occur in this experiment.

Figure 5 shows a high-speed photographic sequence obtained for an experiment from test series 2 (see Section 3.3) by using specimen $2\left(\sigma_{c}=2.8 \mathrm{MPa}\right.$, total areal mass $m_{F}=42.3 \mathrm{kgm}^{-2}$, see Table 1 ) for the outer skin. Soon after the shock wave ( $p_{0}=11.6 \mathrm{MPa}$, $\theta=0.14 \mathrm{~ms}$ ) has reached the sandwich specimen (Fig. 5a) the front face sheet is set in motion and cavitation bubbles emerge in the front water column, very close to the fluid-structure interface, as shown in Fig. 5b. Theoretical models [4] predict that two breaking fronts (BF) emerge at the point of first cavitation, propagating in opposite directions and opening a pool of cavitated water, as observed in the experiment (Fig. 5b). The BF approaching the sandwich plate reaches the fluid-structure interface while the opposite BF continues propagating away from the structure. Subsequently, the front face sheet decelerates due to the support offered by the foam core and the cavitation front contiguous to the fluid-structure interface starts propagating away from the structure, becoming a closing front (CF) and forcing collapse of cavitation bubbles (see Fig. 5c). As time elapses, the bubbles increase in size owing to an increase in strain of the cavitated fluid consequent to continued displacement of the front face sheet. The CF continues propagating and eventually leaves the field of observation, as observed in Fig. 5d. 


\subsubsection{Unsupported inner hull $\left(m_{B} \approx m_{F}\right)$}

We proceed to present high-speed photographs for experiments in which the inner hull specimen was free to slide in the shock tube, i.e. mimicking blast loading of double hulls with $m_{B} \approx m_{F}$. Fig. 6 illustrates a sequence of high-speed photographs recorded in an experiment performed for test series 3 (see Section 3.3) by using monolithic specimens for both outer skin $\left(m_{F}=43.0 \mathrm{kgm}^{-2}\right)$ and inner hull $\left(m_{B}=80.2 \mathrm{kgm}^{-2}\right)$. In frame (a) the incident shock wave ( $p_{0}=12.0 \mathrm{MPa}, \theta=0.14 \mathrm{~ms}$ ) reaches the outer skin and the ensuing motion of this skin results in a pressure wave radiated from its back face, sweeping the water layer (thickness $D=60 \mathrm{~mm}$ ) and impinging on the wet surface of the unsupported inner hull. As a consequence, the inner hull specimen is set in motion and a zone of cavitated water spreads from the fluid-structure interface into the water layer by propagation of a BF, as seen in Fig. 6b. Upon arrival of this BF at the back face of the outer skin, the pressure at this interface vanishes, leading to a rapid increase in plate velocity due to loading of the outer skin front face by the incident shock wave. This, in turn, results in a tensile rarefaction wave radiated from the front interface and travelling into the fluid, giving rise to cavitation in the front water column, as illustrated in Fig. 6c. Subsequently, both specimens slowly decelerate due to a combination of air resistance and frictional forces, causing closing fronts (CFs) to emerge at their front interfaces, both of which propagating into the cavitated water and forcing collapse of the cavitation bubbles which have strongly increased in size as a result of increased strain in the cavitated fluid, see Fig. 6d. Both CFs continue propagating and, as time elapses, the cavitation zone in the front water column has contracted beyond the field of observation, while a gap of cavitated water forms at the back interface of the outer skin plate which slowly collapses by propagation of the CF (see Fig. 6e). 
The high-speed photographic sequence illustrated in Fig. 7 shows the system response observed in an experiment of test series 3, conducted under similar conditions as the latter experiment (Fig. 6) but with the monolithic outer skin specimen replaced by a sandwich plate (specimen $1, \sigma_{c}=1.1 \mathrm{MPa}$, see Table 1 ) of approximately equal mass, $m_{F}=42.5 \mathrm{kgm}^{-2}$. Upon the impact of the incident pressure wave ( $p_{0}=11.5 \mathrm{MPa}, \theta=0.14 \mathrm{~ms}$ ) on the sandwich plate (see Fig. 7a), the front face sheet rapidly acquires a sufficiently high velocity to trigger cavitation in the water, in proximity of the front face sheet; a cavitated pool of water expands by propagation of two BFs (see Fig. 7b), as predicted by [4]. The BF approaching the structure quickly reaches the fluid-structure interface (Fig. 7b), inverts its direction of motion and becomes a CF, propagating away from the interface and forcing the cavitation zone to contract, as seen in Fig. 7c. The acceleration of the rigid inner hull specimen is not sufficient to initiate cavitation in the water layer. However, while the cavitation zone in the front water entirely collapses in the field of observation, a thin gap of cavitated water forms at the interface between the sandwich outer skin and the water layer, due to motion of the inner hull (see Fig. 7d).

\subsection{Structural response}

Having described the cavitation processes observed in the experiments, we now proceed to analyse the response of outer skin and inner hull specimens. We begin by noting that the intensity of structural loading consequent to blast loading is quantified by the specific impulse (impulse per unit area) imparted to the structure. Upon neglecting frictional forces between Orings and tube wall, the impulse applied on the inner hull specimen is given by

$$
I_{B}(t)=\int_{0}^{t} p_{\mathrm{B}, \mathrm{f}}(\tau) \mathrm{d} \tau=m_{B} v_{B}(t)
$$

where $p_{\mathrm{B}, \mathrm{f}}(\tau)$ denotes the pressure time history applied dynamically on the front face of the inner hull. 
In the experiments, $p_{\mathrm{B}, \mathrm{f}}(\tau)$ was measured using the pressure transducer mounted on the inner hull specimen (see Fig. 2a), allowing evaluation of eq.(7).

In the following we present time histories of non-dimensional impulse $\bar{I}_{B}(t)=I_{B}(t) /\left(2 p_{0} \theta\right)$ (measured as described above) and core compression $\varepsilon_{c}(t)$ (deduced from the obtained highspeed photographs) for selected experiments performed with both stationary and unsupported inner hull specimens and we compare the measurements to the corresponding FE predictions.

\subsubsection{Stationary inner hull $\left(m_{B} \rightarrow \infty\right)$}

Measurements and FE predictions of time histories $\bar{I}_{B}(t)$ obtained from test series 2 ( $p_{0}=11.8 \pm 0.2 \mathrm{MPa}, \theta=0.14 \mathrm{~ms}, D=120 \mathrm{~mm}$, see Section 3.3) are presented in Fig. 8a. It can be seen from this figure that both the peak impulse $\bar{I}_{\mathrm{B}, \max }=\max _{t \rightarrow \infty}\left[\bar{I}_{\mathrm{B}}(t)\right]$ as well as the rate at which the impulse $\bar{I}_{B}(t)$ increases (equal to the dynamic pressure) are dramatically reduced when the sandwich construction is employed; these reductions are more pronounced for cores of lower collapse strength $\sigma_{c}$. For e.g. specimen $1\left(\sigma_{c}=1.1 \mathrm{MPa}\right)$ we report $\bar{I}_{\mathrm{B}, \max } \approx 0.4$, a reduction in impulse of more than $60 \%$ in comparison to a rigid plate of equal mass $\left(\bar{I}_{\mathrm{B}, \max } \cong 1\right)$. The experimental measurements of $\bar{I}_{B}(t)$ are adequately captured by the FE calculations. It is interesting to note that the $\bar{I}_{B}(t)$ histories of sandwich specimens with lower compressive strength $\sigma_{c}$, i.e. specimens 1 and 2, exhibit multiple plateaus before reaching $\bar{I}_{\mathrm{B}, \max }$, indicating multiple cycles of nucleation and collapse of a cavitation zone at the front face of the inner hull. However, the short duration of these cavitation phenomena does not permit for significant strain to develop in the cavitated fluid; therefore, cavitation bubbles could not be observed in the experiment. 
The corresponding measurements and predictions of the compressive core strain histories $\varepsilon_{c}(t)$ are presented in Fig. 8b. All types of core investigated here show similar trends: $\varepsilon_{c}(t)$ quickly reaches a peak value and subsequently decreases modestly, owing to elastic springback. Now compare Figs. 8a and 8b: the softest core, i.e. specimen 1, achieved the largest reductions in impulse $\bar{I}_{\mathrm{B} \text {,max }}$, but at the expense of increased permanent core compression $\varepsilon_{c}^{p}$. However, the values of $\varepsilon_{c}^{p}$ are relatively low in the experiments reported here and the $\mathrm{FE}$ predictions confirm this.

\subsubsection{Unsupported inner hull $\left(m_{B} \approx m_{F}\right)$}

In Fig. 9a, measurements and FE predictions of impulse histories $\bar{I}_{B}(t)$ are presented for an experiment performed within test series 3 by using monolithic unsupported specimens for both outer skin and inner hull ( $D=60 \mathrm{~mm}$, see Section 3.3); in addition, FE predictions of the non-dimensional impulse $\bar{I}_{F}(t)=I_{F}(t) /\left(2 p_{0} \theta\right)$ imparted to the outer skin are included in this figure (the experimental apparatus [25] does not allow measuring $I_{F}(t)$ in the presented experiments). High-speed photographs recorded in this experiment are shown in Fig. 6. The upper horizontal line in Figs. 9a and 9b represent analytical predictions [13] of the impulse applied on the outer skin for the case of infinite water layer thickness, $D \rightarrow \infty$, while the lower horizontal line denotes predictions [13] of the impulse applied on the inner hull alone, with the outer skin structure absent, i.e. $m_{F}=0$. It can be seen that $\bar{I}_{F}(t)$ rapidly rises consequent to shock loading and this impulse is partly transmitted into the water layer, causing a rise in $\bar{I}_{B}(t)$ at a later time. Neglecting frictional forces, this impulse is entirely converted into momentum (eq. (7)), resulting in acceleration of the inner hull specimen and giving rise to cavitation at its fluid-structure interface. Consequently, loading of the inner hull ceases and the imparted impulse reaches a peak value at this point, $\bar{I}_{\mathrm{B}, \max }$. Similarly, $\bar{I}_{F}(t)$ 
culminates at the instant when the water cavitates at both sides of the outer skin specimen (recall Fig. 6c), with the peak value $\bar{I}_{\mathrm{F}, \max }$ well below the rigid plate predictions, $D \rightarrow \infty$.

Measurements and FE predictions of $\bar{I}_{B}(t)$ are found in excellent agreement and show that the peak impulse $\bar{I}_{\mathrm{B}, \max }$ slightly exceeds the predictions $m_{F}=0$. This can be explained by recalling that the inner hull is loaded by a pressure wave radiated from the back face of the outer skin. Such wave has significant rise time owing to the inertia of the outer skin and this can lead to delayed onset of cavitation and to more severe loading.

Similar information is shown in Fig. 9b for an experiment conducted under similar conditions as the latter experiment (Fig. 9a) but with the monolithic outer skin specimen replaced by a sandwich plate of approximately equal mass (specimen $1, \sigma_{c}=1.1 \mathrm{MPa}$, see Table 1 ); a highspeed photographic sequence of this experiment is presented in Fig. 7. The outer skin reads a sharp rise in $\bar{I}_{F}(t)$ consequent to the shock wave impact, soon after which a bend in the $\bar{I}_{F}(t)$ curve occurs due to the emergence of cavitation very close to the front face sheet (see Fig. $7 \mathrm{~b})$. The impulse $\bar{I}_{F}(t)$ continues to rise at a relatively high rate owing to the rapid collapse of the cavitated region by propagation of a CF, as shown in the high-speed photograph of Fig. 7c. These observations are qualitatively consistent with the findings of previous studies on underwater blast loading of sandwich plates [5, 12] which support the conclusion that the efficiency of impulse transfer to the plate is increased when a layer of water remains at the fluid-structure interface subsequent to first cavitation. As shown in Fig. 7, the velocity of the inner hull specimen was not sufficiently high to initiate a BF in the water layer and, as a consequence, the impulse $\bar{I}_{B}(t)$ increases stepwise (see Fig. 9b) due to pressure waves travelling in the water layer, similar to what observed from Fig. 8a. Now 
compare the peak values $\bar{I}_{\mathrm{F} \text {,max }}$ and $\bar{I}_{\mathrm{B} \text {,max }}$ between Figs. 9a and 9b: sandwich construction leads to significant reductions of both $\bar{I}_{\mathrm{F}, \max }$ and $\bar{I}_{\mathrm{B}, \max }$ compared to monolithic designs of equal mass. Again, good correlation was achieved between FE predictions and measurements.

\subsection{Sensitivity of imparted impulse and core crush to the water layer thickness}

We now proceed to explore the effect of the water layer thickness $D$ upon the core crush $\varepsilon_{c}^{p}$ as well as on the peak impulses imparted to the outer skin and inner hull, $\bar{I}_{\mathrm{F} \text {,max }}$ and $\bar{I}_{\mathrm{B} \text {,max }}$, respectively, for both stationary and unsupported inner hulls.

\subsubsection{Stationary inner hull $\left(m_{B} \rightarrow \infty\right)$}

Figure 10 presents measurements and predictions of $\bar{I}_{\mathrm{B}, \max }$ and $\varepsilon_{c}^{p}$ obtained from test series 1 ( $p_{0}=12 \pm 0.5 \mathrm{MPa}, \theta=0.14 \mathrm{~ms}$ ) as functions of the water layer thickness $D$, together with the corresponding FE predictions. Both predictions and measurements show that regardless whether the outer skin is monolithic or made from a sandwich plate, both $\bar{I}_{\mathrm{B} \text {, max }}$ and $\bar{I}_{\mathrm{F} \text {,max }}$ are only weakly sensitive to variations of $D$, see Fig. 10a. The FE predictions also show that the discrepancies between $\bar{I}_{\mathrm{F}, \max }$ and $\bar{I}_{\mathrm{B}, \max }$ are insignificant for both monolithic and sandwich hulls. In addition, Fig. 10a underpins the findings drawn from Fig. 8: sandwich construction of the outer skin remarkably mitigates the severity of structural loading in a blast event compared to a monolithic plate of equal mass. A relatively low degree of core crush $\varepsilon_{c}^{p} \cong 0.1$ is sufficient to achieve large benefits in terms of blast performance, see Fig. 10b. 


\subsubsection{Unsupported inner hull $\left(m_{B} \approx m_{F}\right)$}

In Fig. 11, measurements of $\bar{I}_{\mathrm{B}, \max }$ obtained from test series $3\left(p_{0}=11.7 \pm 0.3 \mathrm{MPa}\right.$, $\theta=0.14 \mathrm{~ms}$, see Section 3.3) are plotted against the water layer thickness $D$, for the case of (a) monolithic double hulls and (b) sandwich double hulls (specimen 1, $\sigma_{c}=1.1 \mathrm{MPa}$, see Table 1); in addition we include in both Figs. 11a and 11b the dynamic FE predictions of $\bar{I}_{\mathrm{F} \text {,max }}$ and $\bar{I}_{\mathrm{B} \text {, max }}$ as well as analytical rigid plate predictions [13] for the cases $D \rightarrow \infty$ and $m_{F}=0$, respectively. Figure 12 a shows that upon increasing $D$ of a monolithic double hull, $\bar{I}_{\mathrm{F}, \max }$ modestly increases and approaches the predictions $D \rightarrow \infty$, as expected. The dependence of $\bar{I}_{\mathrm{B} \text {, max }}$ on the water layer thickness $D$ is only marginal, with values of $\bar{I}_{\mathrm{B} \text {, max }}$ approximately equal to the predictions $m_{F}=0$. The measurements of $\bar{I}_{\mathrm{B} \text {, max }}$ are adequately predicted by the FE simulations.

Similarly, for the case of sandwich double hulls, Fig. 11b shows that an increase in $D$ leads to an increase of $\bar{I}_{\mathrm{F}, \max }$ while the values of $\bar{I}_{\mathrm{B}, \max }$ slightly decrease, with both $\bar{I}_{\mathrm{B} \text {, max }}$ and $\bar{I}_{\mathrm{F} \text {, max }}$ significantly lower than those associated to blast loading of monolithic double hulls, see Fig. 11a. Note that the core crush $\varepsilon_{c}^{p}$ measured in these experiments was less than $15 \%$.

\section{CONCLUSIONS}

This study has investigated the 1D response to underwater blast loading of double-walled hulls comprising of a monolithic inner pressure hull, an outer skin and a water layer sandwiched between these. Laboratory scale underwater shock experiments were performed using a previously developed transparent shock tube apparatus [25] and high-speed photography was employed to observe structural motion as well as the ensuing cavitation phenomena initiated by FSI. Measurements of impulse and core crush were found in good 
agreement with the predictions obtained from detailed FE simulations. The experimental results give valuable insight into the cavitation process and provide practical guidelines for the design of double-walled hulls resistant to underwater blast. The main findings of this study are as follows:

- For double hulls composed of a monolithic outer skin and a stationary inner hull, no cavitation is observed in the water layer and the entire blast impulse is imparted to the structure. In contrast, when sandwich construction is employed for the outer skin, cavitation processes initiate in the water very close to the front face sheet of the sandwich hull and result in a dramatic reduction of the impulse imparted to the inner hull (-60\% compared to the case of a monolithic outer skin).

- For blast loading of structures comprising a monolithic outer skin and an unsupported inner hull of similar mass, cavitation initiates in the water layer and continues to evolve in the front water, remarkably reducing the impulse imparted to both structures compared to blast loading of a stationary inner hull. The effect of sandwich construction is to locate the onset and evolution of the cavitation process exclusively in the front water, leading to further improvements in blast performance.

- $\quad$ The impulse applied on stationary or unsupported inner hulls in an underwater blast event is only scarcely sensitive to variations in the thickness of the water layer, regardless of the construction of the outer skin. 


\section{Acknowledgements}

The authors would like to thank P.R.Thompson (Weidlinger Associates Ltd, UK), S.Stojko and S.Banks (Rolls-Royce plc, UK), as well as Prof. N.Petrinic (University of Oxford) for stimulating conversations. We are grateful for the financial support from EPSRC and DSTL through grant EP/G042586/1, managed by Prof A.Groves.

\section{References}

[1] R.H. Cole, Underwater Explosions, Princeton University Press, Princeton, NJ, USA, 1948.

[2] M.M. Swisdak, Explosion effects and properties: part II - explosion effects in water., Naval surface weapons centre, Dahlgren, VA, USA, 1978.

[3] G.I. Taylor, The pressure and impulse of submarine explosion waves on plates, in: The scientific papers of G.I. Taylor, Vol III, Cambridge University Press, Cambridge, UK, 1963, pp. 287-303.

[4] E.H. Kennard, Cavitation in an elastic liquid, Phys. Rev., 63 (1943) 172-181.

[5] Y. Liang, A.V. Spuskanyuk, S.E. Flores, D.R. Hayhurst, J.W. Hutchinson, R.M. McMeeking, A.G. Evans, The response of metallic sandwich panels to water blast, J. Appl. Mech., 71 (2007) 81-99.

[6] G.J. McShane, V.S. Deshpande, N.A. Fleck, The underwater blast resistance of metallic sandwich beams with prismatic lattice cores, J. Appl. Mech., 74 (2007) 352-364.

[7] X. Qiu, V.S. Deshpande, N.A. Fleck, Finite element analysis of the dynamic response of clamped sandwich beams subject to shock loading, Eur. J. Mech. A-Solids, 22 (2003) 801-814.

[8] Z. Xue, J.W. Hutchinson, A comparative study of impulse-resistant metal sandwich plates, Int. J. Impact Eng., 30 (2004) 1283-1305.

[9] N.A. Fleck, V.S. Deshpande, The resistance of clamped sandwich beams to shock loading, J. Appl. Mech., 71 (2004) 386-401.

[10] V.S. Deshpande, N.A. Fleck, One-dimensional response of sandwich plates to underwater shock loading, J. Mech. Phys. Solids, 53 (2005) 2347-2383.

[11] J.W. Hutchinson, Z. Xue, Metal sandwich plates optimized for pressure impulses, Int. J. Mech. Sci., 47 (2005) 545-569.

[12] R.M. McMeeking, A.V. Spuskanyuk, M.Y. He, V.S. Deshpande, N.A. Fleck, A.G. Evans, An analytical model for the response to water blast of unsupported metallic sandwich panels, Int. J. Solids Struct., 45 (2007) 478-496.

[13] A. Schiffer, V.L. Tagarielli, N. Petrinic, A.F.C. Cocks, The Response of Submerged Rigid Plates to Blast Loading: Analytical Models and Finite Element Predictions, J. Appl. Mech., (2011).

[14] US Office of Naval Research, Underwater explosion research: a compendium of British and American reports, Washington, DC, USA, 1950.

[15] D.J. Hall, Examination of the effects of underwater blasts on sandwich composite structures, Compos. Struct., 11 (1989) 101-120.

[16] A.P. Mouritz, D.S. Saunders, S. Buckley, The damage and failure of GRP laminates by underwater explosion shock loading, Composites, 25 (1994) 431-437.

[17] K. Ramajeyathilagam, C.P. Vendhan, Deformation and rupture of thin rectangular plates subjected to underwater shock, Int. J. Impact Eng., 30 (2004) 699-719. 
[18] V.S. Deshpande, A. Heaver, N.A. Fleck, An underwater shock simulator, P. Roy. Soc. Lond. A Mat., 462 (2006) 1021-1041.

[19] G.J. McShane, V.S. Deshpande, N.A. Fleck, Underwater blast response of free-standing sandwich plates with metallic lattice cores, Int. J. Impact Eng., 37 (2010) 1138-1149.

[20] H.D. Espinosa, S. Lee, N. Moldovan, A novel fluid structure interaction experiment to investigate deformation of structural elements subjected to impulsive loading, Exp. Mech., 46 (2006).

[21] L.F. Mori, S. Lee, Z.Y. Xue, A. Vaziri, D.T. Queheillalt, K.P. Dharmasena, H.N.G. Wadley, J.W. Hutchinson, H.D. Espinosa, Deformation and fracture modes of sandwich structures subjected to underwater impulsive loads, J. Mech. Mater. Struct., 2 (2007) 1981-2006.

[22] L.F. Mori, D.T. Queheillalt, H.N.G. Wadley, H.D. Espinosa, Deformation and failure modes of I-core sandwich structures subjected to underwater impulsive loads, Exp. Mech., 49 (2009) 257-275.

[23] F. Latourte, D. Gregoire, D. Zenkert, X. Wei, H.D. Espinosa, Failure mechanisms in composite panels subjected to underwater impulsive loads, J. Mech. Phys. Solids, 59 (2011) 1623-1646.

[24] H. Wadley, K. Dharmasena, Y. Chen, P. Dudt, D. Knight, R. Charette, K. Kiddy, Compressive response of multilayered pyramidal lattices during underwater shock loading, Int. J. Impact Eng., 35 (2008) 1102-1114.

[25] A. Schiffer, V. Tagarielli, L., The response of rigid plates to blast in deep water: fluidstructure interaction experiments, P. Roy. Soc. Lond. A Mat., 468 (2012) 2807-2828.

[26] A. Schiffer, V.L. Tagarielli, One-Dimensional Response of Sandwich Plates to Underwater Blast: Fluid-Structure Interaction Experiments and Simulations, J. Mech. Phys. Solids, (2013) submitted.

[27] S. Arezoo, V.L. Tagarielli, N. Petrinic, J.M. Reed, The mechanical response of Rohacell foams at different length scales, J. Mater. Sci., 46 (2011) 6863-6870.

[28] Abaqus, Analysis User's Manual 6.8, Dassault Systemes Simulia Corp., 2008.

[29] V.S. Deshpande, N.A. Fleck, Isotropic Constitutive Model for Metallic Foams, J. Mech. Phys. Solids, 48 (2000) 1253-1276.

[30] S. Arezoo, V.L. Tagarielli, C.R. Siviour, N. Petrinic, Compressive deformation of Rohacell foams: Effects of strain rate and temperature, Int. J. Impact Eng., 51 (2013) 5057.

[31] D.D. Radford, V.S. Deshpande, N.A. Fleck, The use of metal foam projectiles to simulate shock loading on a structure, Int. J. Impact Eng., 31 (2005) 1152-1171.

[32] V. Tagarielli, L., V.S. Deshpande, N.A. Fleck, Prediction of the dynamic response of composite sandwich beams under shock loading, Int. J. Impact Eng., 37 (2010) 854-864. 


\section{Tables}

Table 1: Foam core properties and dimensions of the sandwich specimens under investigation

\begin{tabular}{cccccccc}
\hline $\begin{array}{c}\text { specimen } \\
\text { number }\end{array}$ & $\begin{array}{c}\text { foam } \\
\text { density } \\
\rho_{c}\left(\mathbf{k g m}^{-3}\right)\end{array}$ & $\begin{array}{c}\text { foam } \\
\text { pore size } \\
\left.d_{\mathrm{p}} \mathbf{( m m}\right)\end{array}$ & $\begin{array}{c}\text { core } \\
\text { diameter }\end{array}$ & $\begin{array}{c}\text { core } \\
\text { thickness }\end{array}$ & $\begin{array}{c}\text { compressive } \\
\text { strength }\end{array}$ & $\begin{array}{c}\text { densification } \\
\text { strain }\end{array}$ & $\begin{array}{c}\text { Young's } \\
\text { modulus }\end{array}$ \\
\hline 1 & 71 & 0.96 & 25.5 & 30 & 1.1 & 0.86 & 100 \\
2 & 110 & 0.93 & 25.5 & 20 & 2.8 & 0.85 & 160 \\
3 & 200 & 0.64 & 26.0 & 15 & 6.5 & 0.83 & 250 \\
\hline
\end{tabular}




\section{Figures}

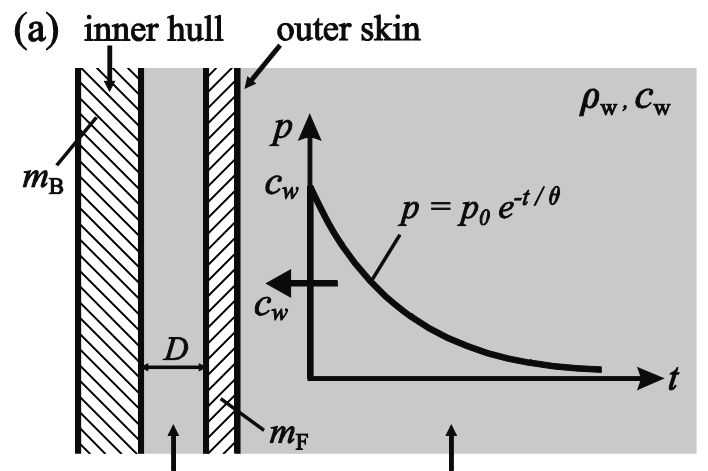

water layer

front water

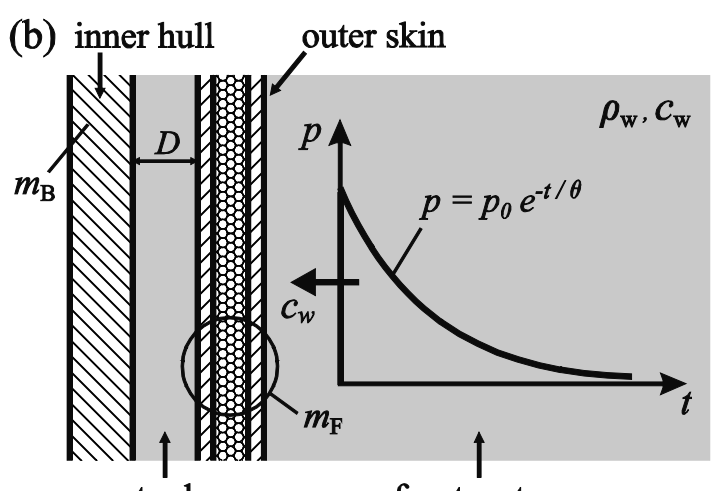

water layer

front water

Fig. 1 Sketches of problem geometry and loading case for (a) monolithic design and (b) sandwich design.

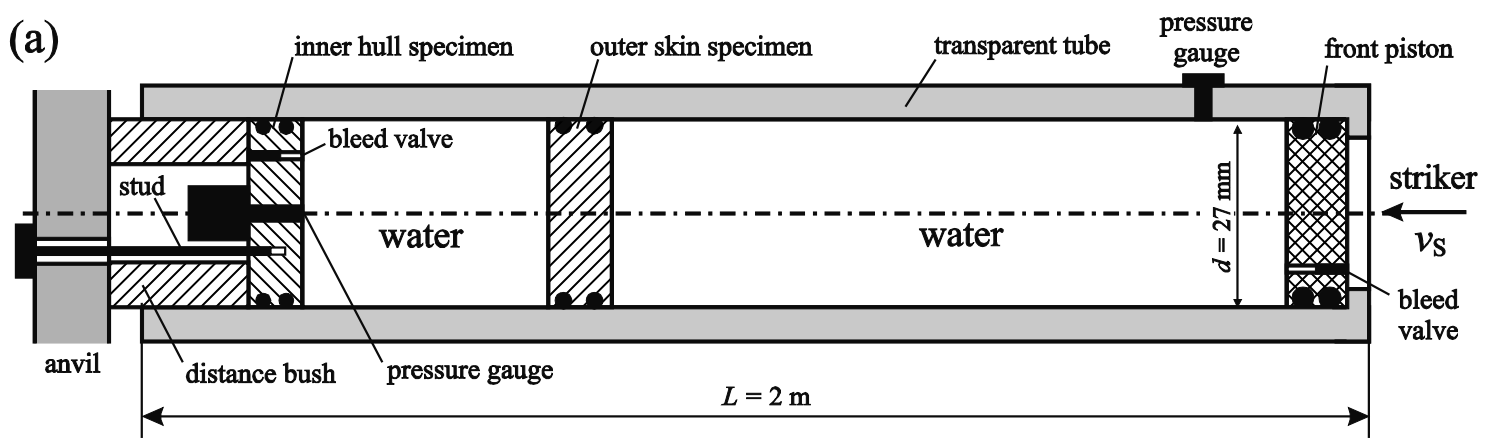

(b)

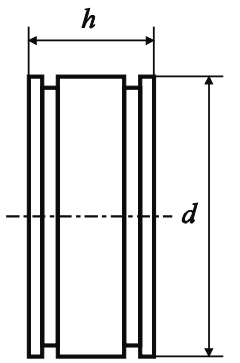

(c)

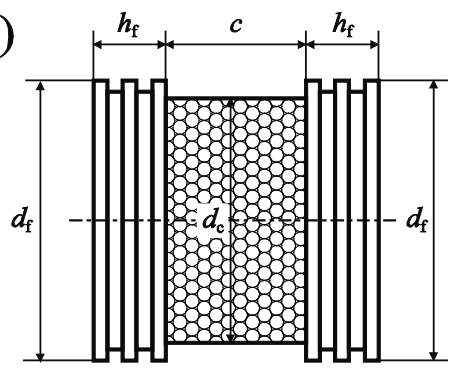

Fig. 2 (a) Schematics of the laboratory setup used for performing 1D underwater blast experiments on a double hull, for the case of a monolithic design with motion of the inner hull constrained. Experiments with unsupported inner hulls are performed with anvil and distance bush absent. (b) Specimen geometry of outer skin plates in monolithic designs and (c) in sandwich design. 


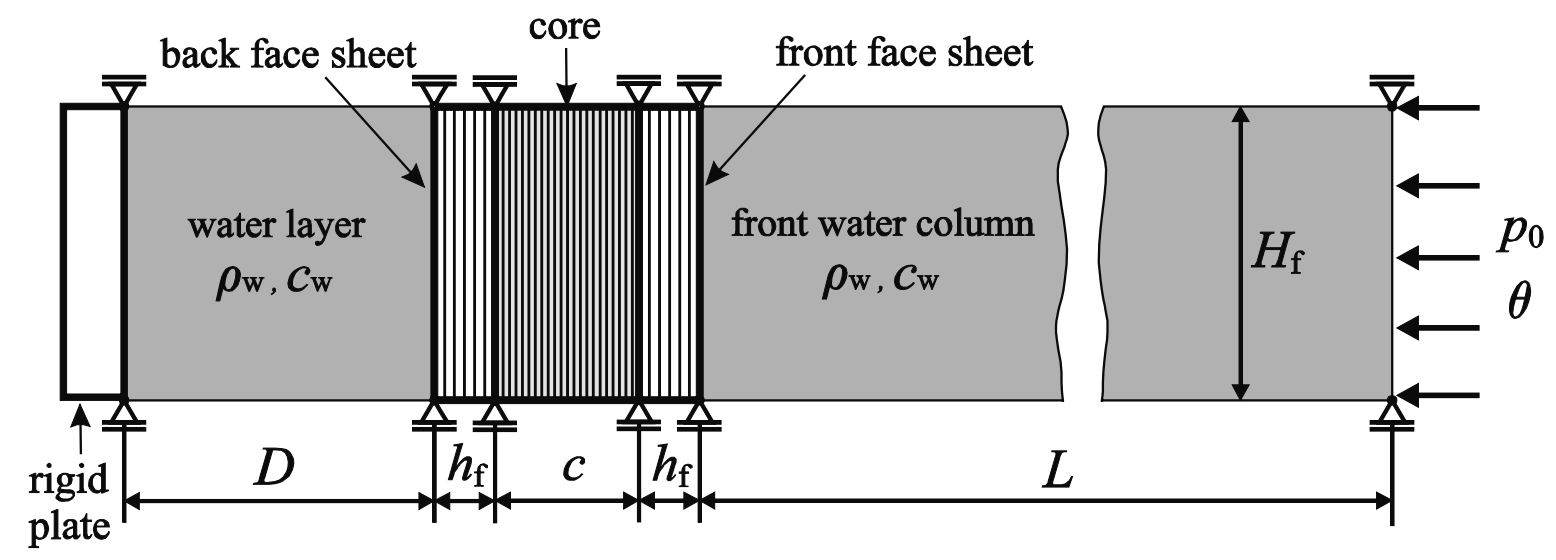

Fig. 3 Schematic of the FE model used to simulate the 1D response of a double hull of sandwich construction, with free-standing monolithic inner hull. The FE models for monolithic double-hulls follow a similar scheme, but with the core and the back face sheet absent.

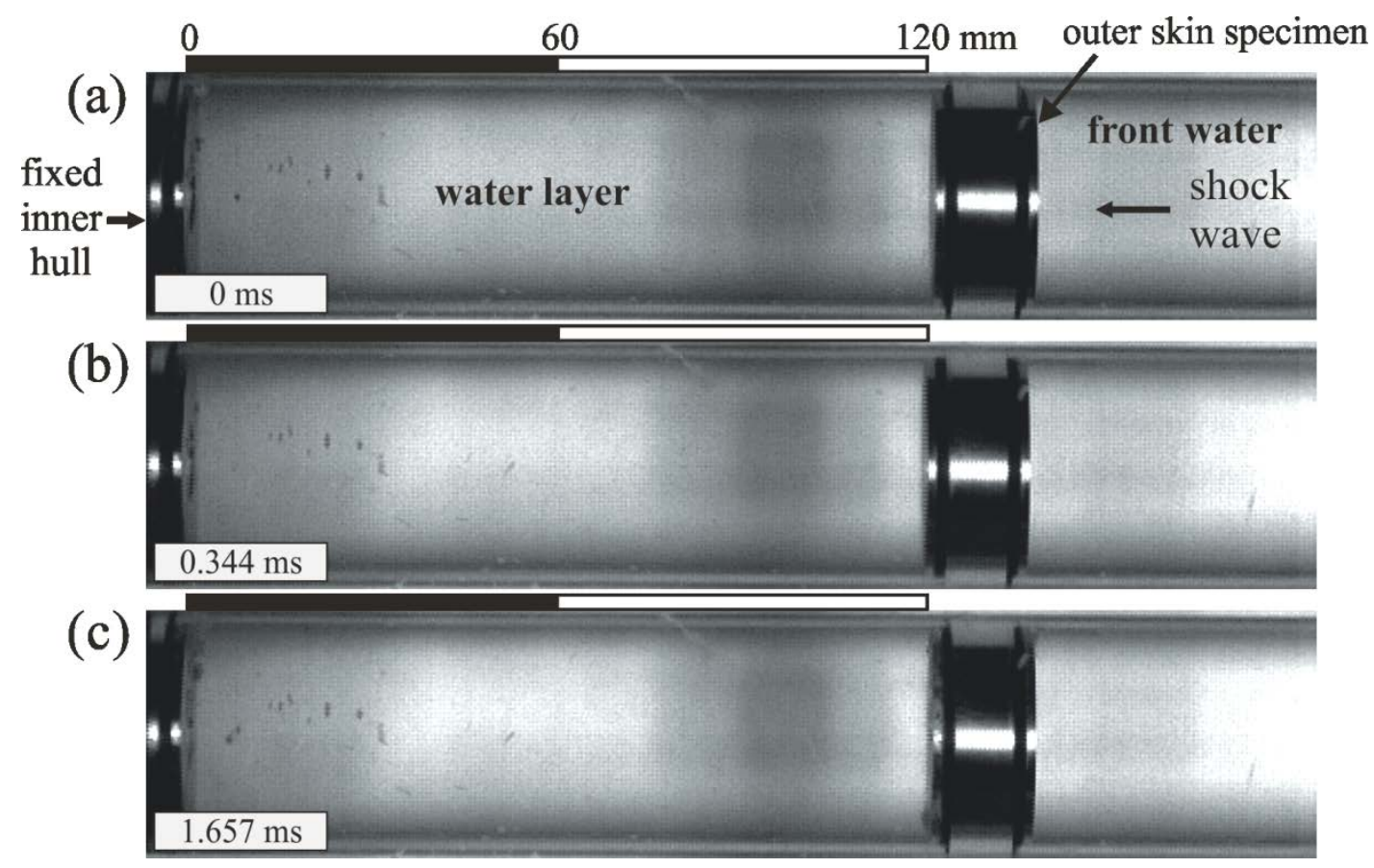

Fig. 4 Sequence of high-speed photographs for an experiment performed for test series 1 showing the response of the monolithic outer skin specimen $\left(m_{F}=43.0 \mathrm{kgm}^{-2}\right)$ consequent to underwater blast loading in the transparent shock tube; $D=120 \mathrm{~mm}, p_{0}=11.6 \mathrm{MPa}, \theta=0.14 \mathrm{~ms}$. 


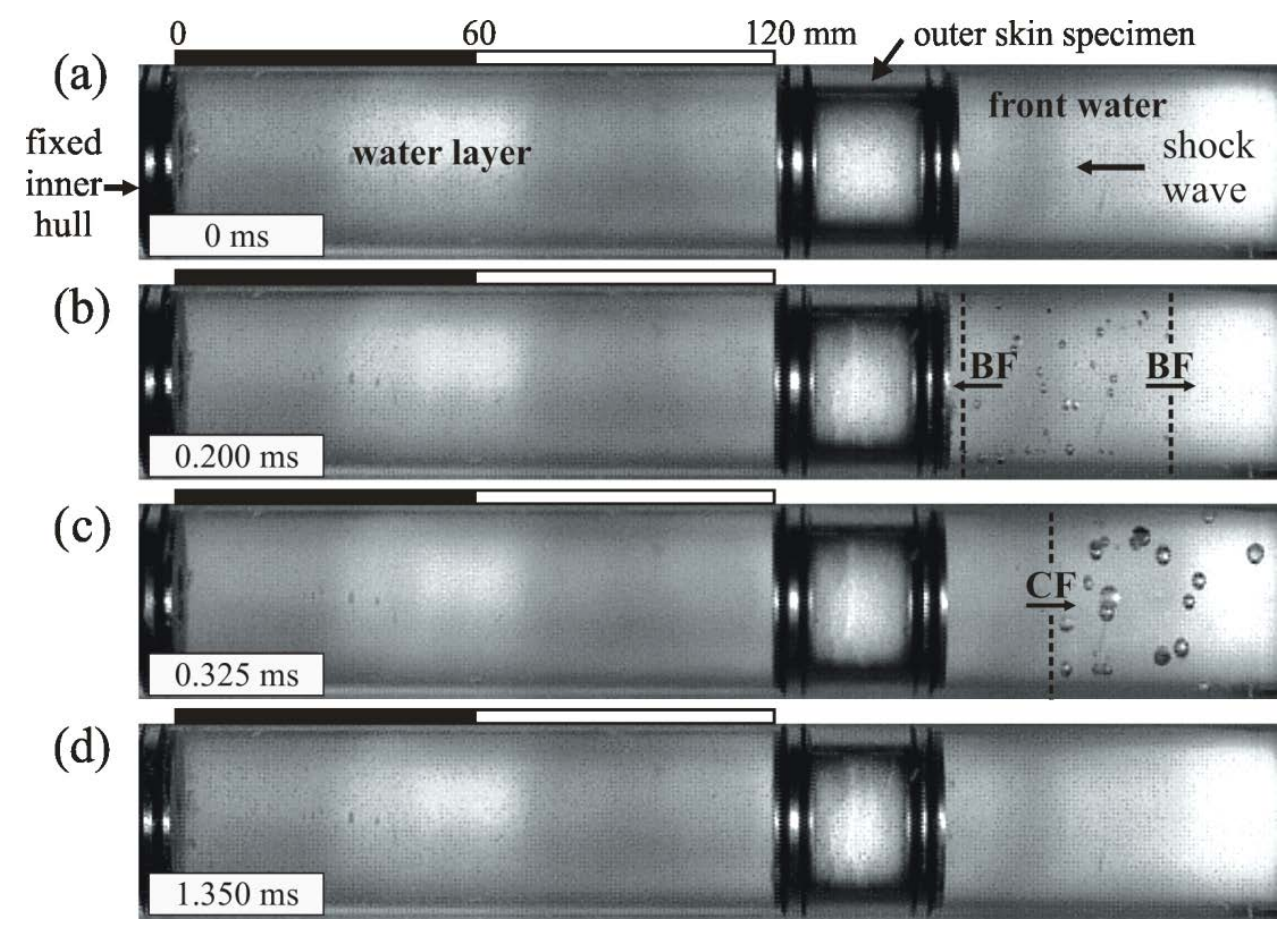

Fig. 5 Sequence of high-speed photographs for an experiment performed for test series 2, showing the blast response of the sandwich specimen (specimen 2, $m_{F}=42.3 \mathrm{kgm}^{-2}$ ) as well as the propagation of breaking fronts (BFs) and closing fronts (CFs); $D=120 \mathrm{~mm}, p_{0}=11.6 \mathrm{MPa}, \theta=0.14 \mathrm{~ms}$.

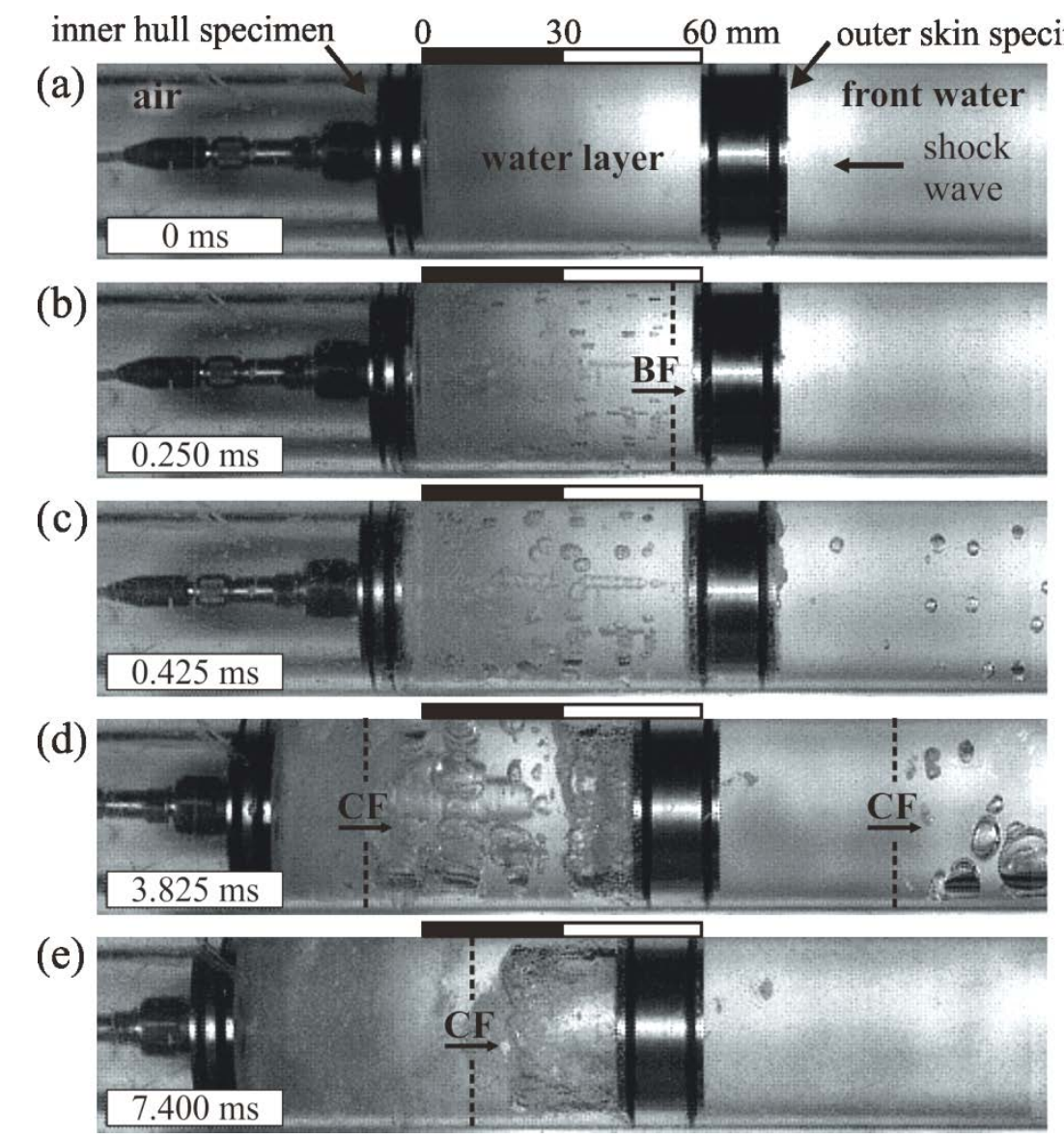

Fig. 6 Sequence of high-speed photographs for an experiment performed for test series 3, showing the blast response of the monolithic specimens $\left(m_{F}=43.0 \mathrm{kgm}^{-2}, m_{B}=80.2 \mathrm{kgm}^{-2}\right)$ as well as the propagation of breaking fronts (BFs) and closing fronts (CFs); $D=60 \mathrm{~mm}, p_{0}=12.0 \mathrm{MPa}, \theta=0.14 \mathrm{~ms}$. 


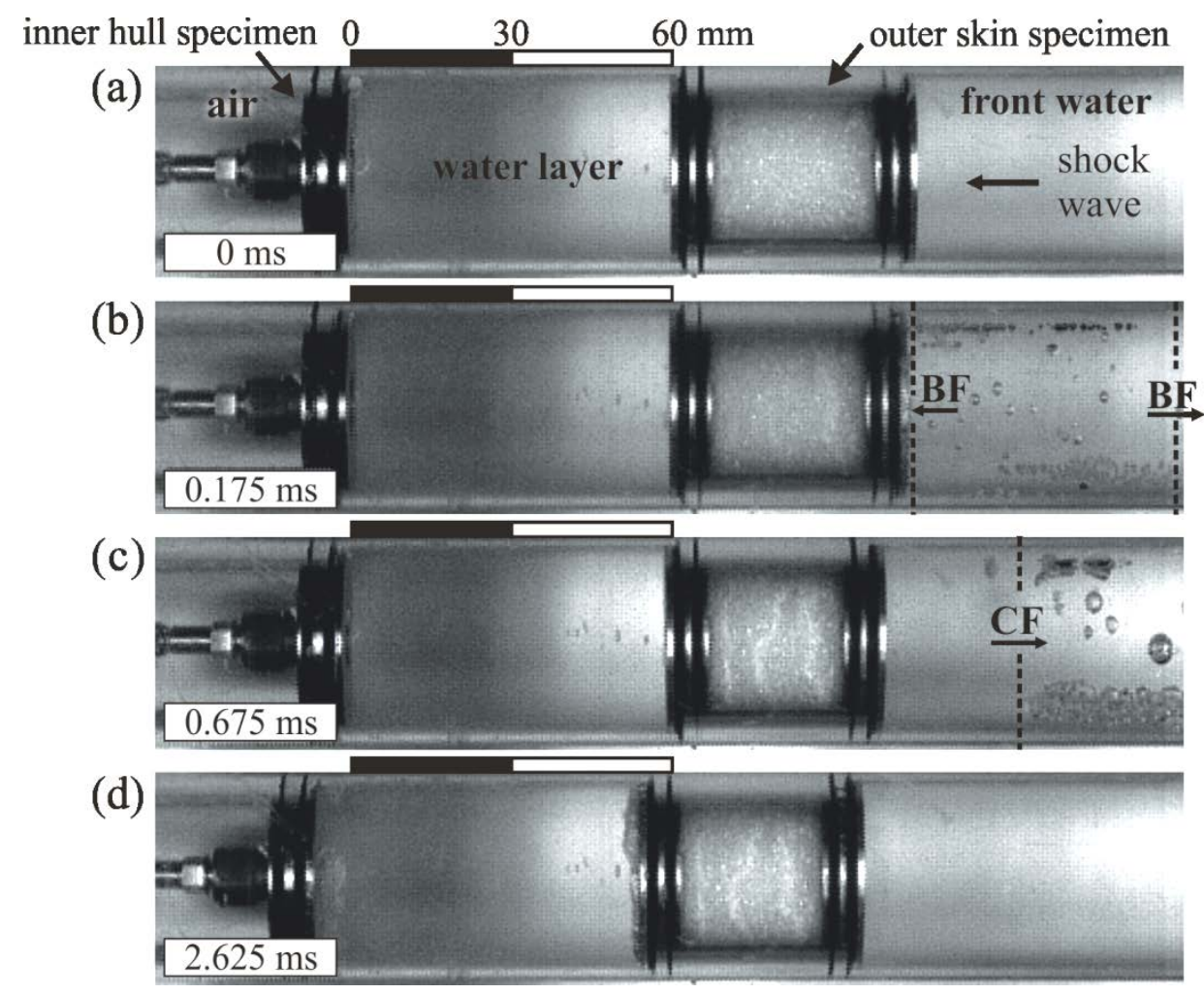

Fig. 7 Sequence of high-speed photographs for an experiment performed for test series 3, showing the blast responses of both the monolithic inner hull $\left(m_{B}=80.2 \mathrm{kgm}^{-2}\right)$ and the outer sandwich hull (specimen 1, $m_{F}=42.5 \mathrm{kgm}^{-2}$ ) as well as the propagation of breaking fronts (BFs) and closing fronts (CFs); $D=60 \mathrm{~mm}, p_{0}=11.5 \mathrm{MPa}, \theta=0.14 \mathrm{~ms}$.

(a)

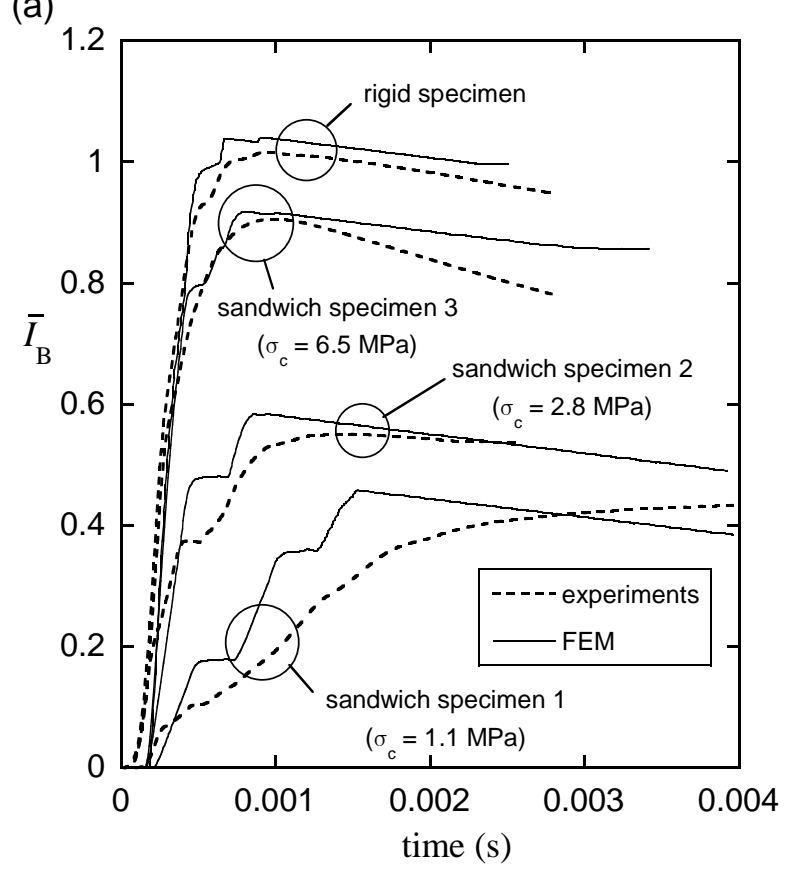

(b)

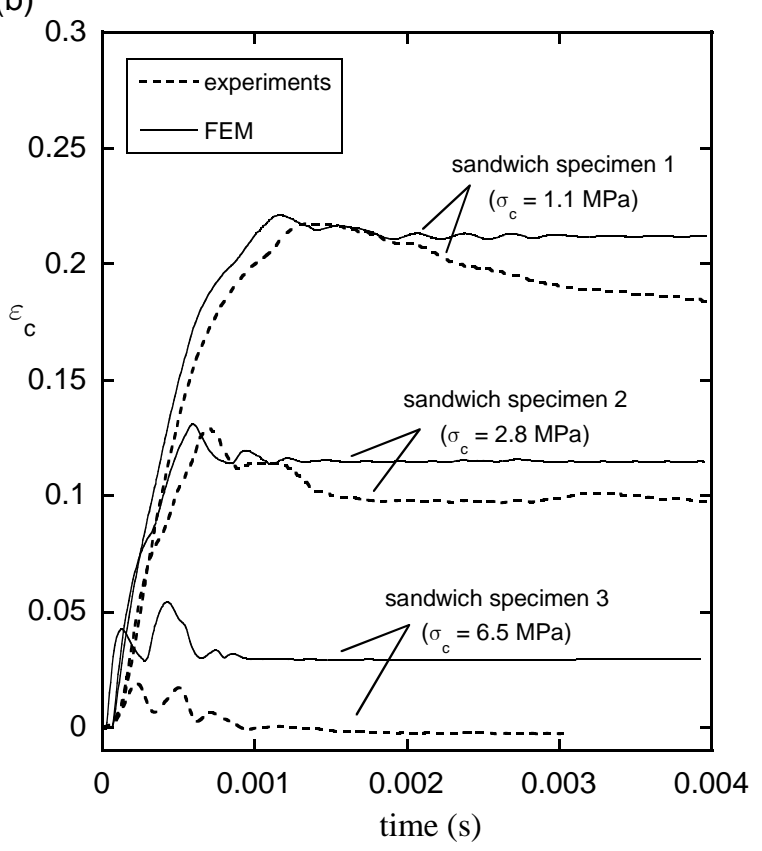

Fig. 8 Stationary inner hull, test series $2\left(p_{0}=11.8 \pm 0.2 \mathrm{MPa}, \theta=0.14 \mathrm{~ms}, D=120 \mathrm{~mm}\right)$ :

(a) Measurements and FE predictions of the impulse histories applied on the inner hull, $\bar{I}_{B}(t)$; sandwich specimens of different core strength are compared with a rigid plate; (b) measured and predicted time histories of the compressive core strain $\varepsilon_{c}(t)$ for the same set of experiments. 
(a)

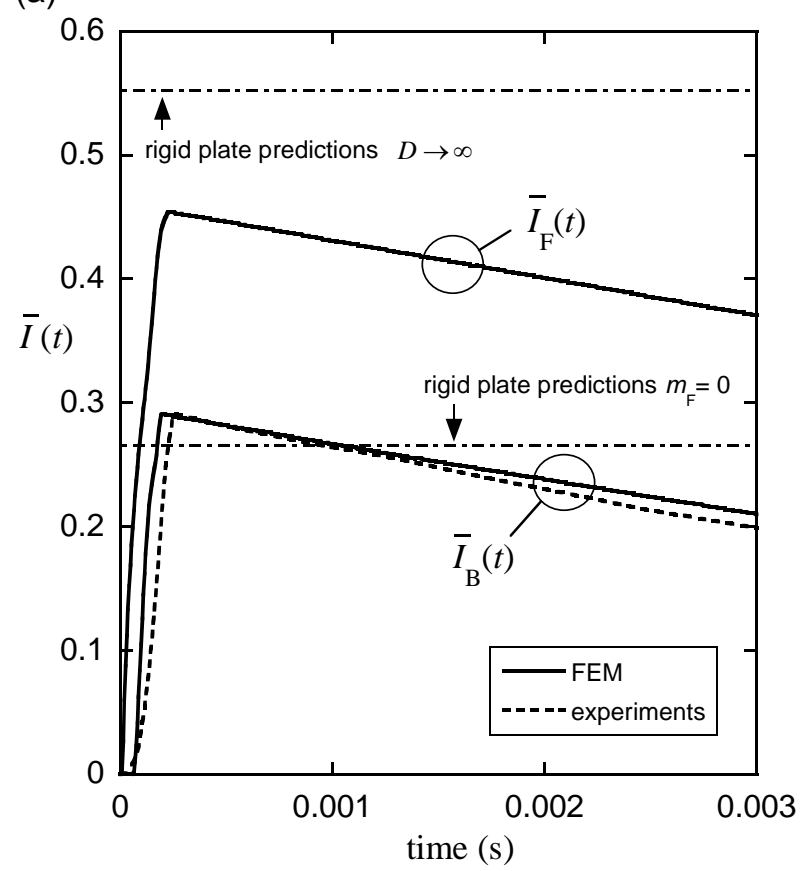

(b)

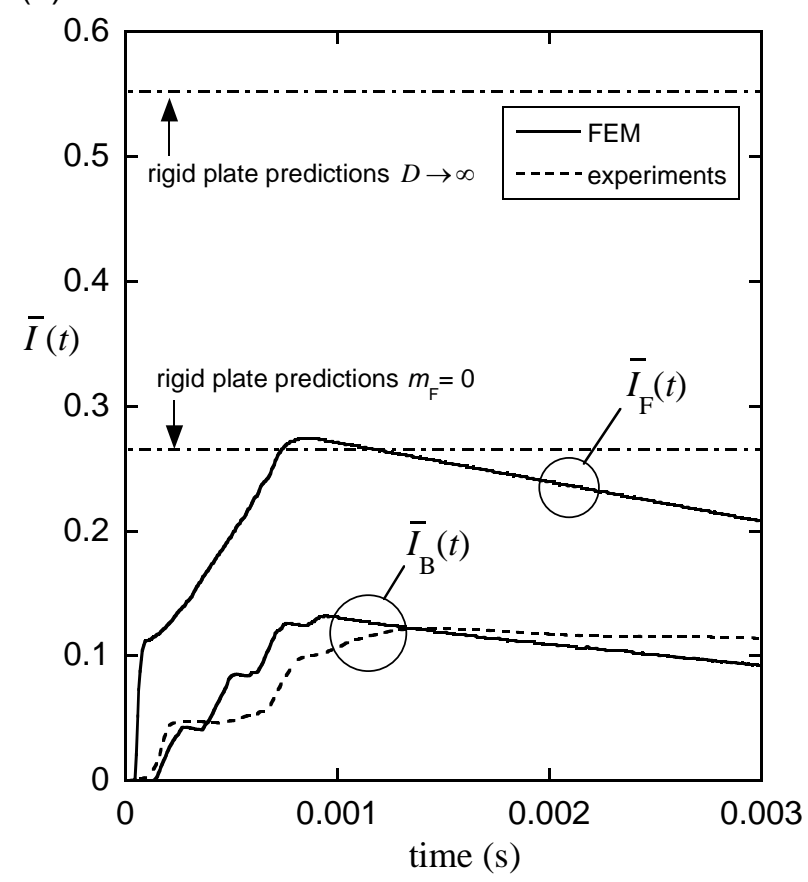

Fig. 9 (a) Measurements and FE predictions of the impulse histories applied on the outer skin and the unsupported inner hull, $\bar{I}_{F}(t)$ and $\bar{I}_{B}(t)$, respectively, consequent to shock loading of a monolithic double hull ( $p_{0}=12.0 \mathrm{MPa}, \theta=0.14 \mathrm{~ms}, D=60 \mathrm{~mm}$ ) and (b) of a sandwich double-hull (specimen 1, $p_{0}=11.5 \mathrm{MPa}, \theta=0.14 \mathrm{~ms}, D=60 \mathrm{~mm}$ ) of equal mass.

(a)

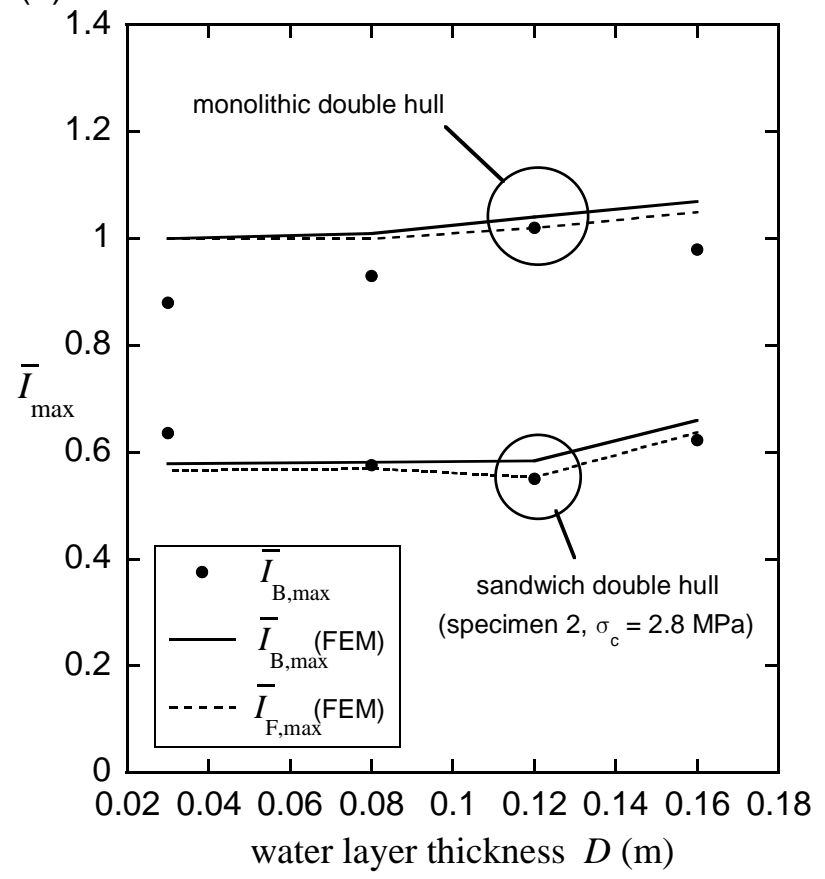

(b)

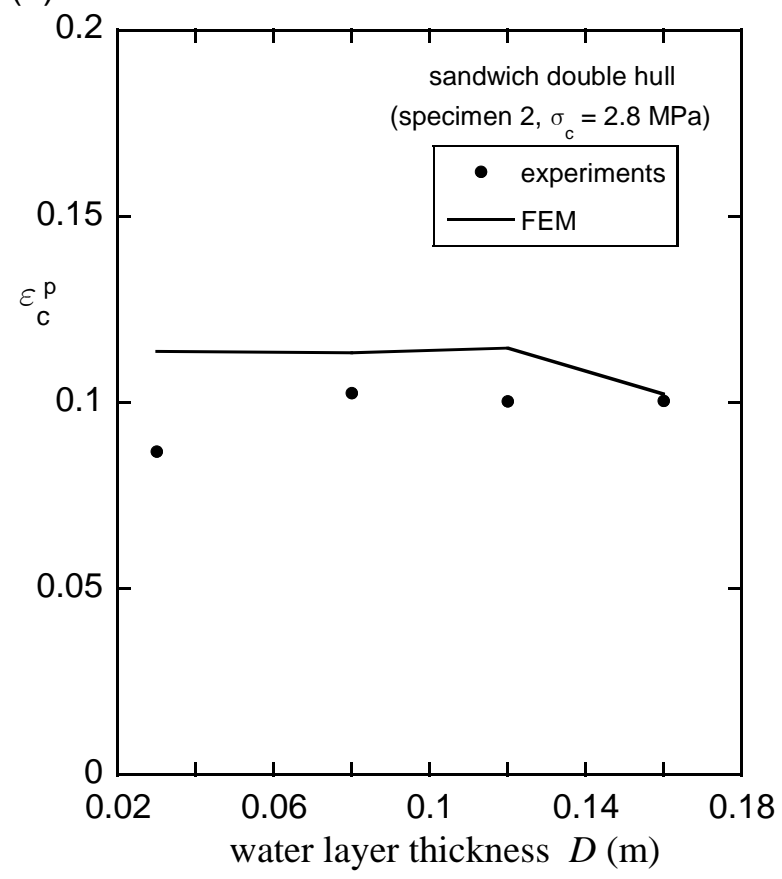

Fig. 10 Test series 1 , stationary inner hull ( $p_{0}=12 \pm 0.5 \mathrm{MPa}, \theta=0.14 \mathrm{~ms}$ ): (a) effect of the water layer thickness $D$ on the maximum impulse imparted to the outer skin and inner hull, denoted as $\bar{I}_{\mathrm{F}, \max }$ and $\bar{I}_{\mathrm{B}, \max }$, respectively; full circles indicate the measurements while the solid and dashed lines represent FE predictions. (b) Measurements and FE predictions of the core crush $\varepsilon_{c}^{p}$ as functions of the thickness $D$. 
(a)

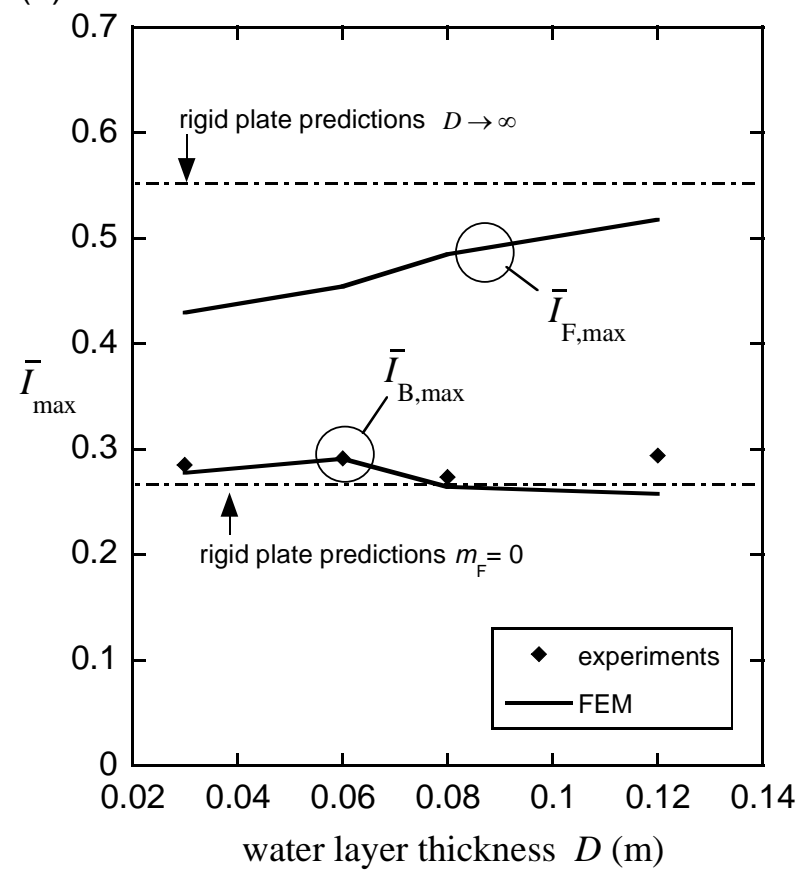

(b)

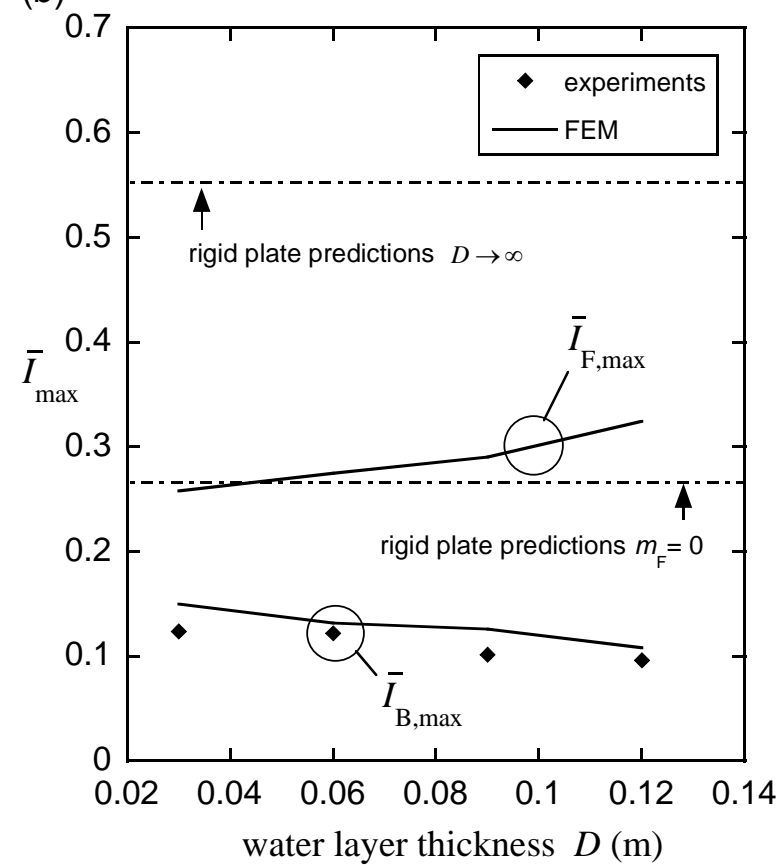

Fig. 11 Test series 3, unsupported inner hull ( $p_{0}=11.7 \pm 0.3 \mathrm{MPa}, \theta=0.14 \mathrm{~ms}$ ): (a) Measurements and FE predictions of $\bar{I}_{\mathrm{F} \text {,max }}$ and $\bar{I}_{\mathrm{B} \text {,max }}$ plotted against the water layer thickness $D$ for experiments performed with monolithic outer skin and inner hull specimens; (b) similar information for experiments in which the monolithic outer skin specimens were replaced by sandwich plates (all of which of specimen 1 type). 